\title{
Fine log interpretation model of reservoir in Huatugou oilfield
}

\author{
Shiyue Fang ${ }^{1}$, Chao Niu ${ }^{1, \mathrm{a}}$, Zhen Liu ${ }^{1}$, Changhong Gao ${ }^{1}$, Shiyue $\mathrm{Tang}^{2}$ and Jukun Chen ${ }^{2}$ \\ ${ }^{1}$ College of Geology and Environment, Xi'an University of Science and Technology,Shaanxi, Xi'an 710054, China \\ ${ }^{2}$ Qinghai oil field, Dunhuang, 736202, China
}

\begin{abstract}
Aiming at some problems exposed in Huatugou oilfield during the development process, such as the imperfect injection-production pattern in some area, the low corresponding rate between oil and injection wells, the low control and producing degree, the poor effect of the water flooding and so on. Starting from the four-property characteristics, establish the fine model of well logging interpretation. The interpretation model has a better precision for obtaining the reservoir parameters. The research results provide a reliable basis for the analysis of the relationship between oil and water, the development evaluation, the adjustment of the injection-production well pattern, play a positive role in the improving on the geological understanding, the increase of the oil production and the level of development in the Huatugou oil field.
\end{abstract}

Keywords: Huatugou oilfield; reservoir; log interpretation model; four-property relationship.

\section{Introduction}

Huatugou oilfield belongs to the two different types of main oil deposit which are the structural trap and lithologic structural composite trap. The main feature is the low resistivity, the serious heterogeneity, and the complex relationship between oil and water of the formation. Therefore, it is very important to use the large number of the well logging data, give the fine interpretation of the reservoir physical parameters in this area, and determine the reservoir porosity, water saturation in the oil layer, which can help us carry out a fine description of the oil layer, build the geological model, simulate the reservoir, calculate the reserves and choose a favorable development district.

For the purpose to obtain the core analysis data and oil testing data of the target strata, well log interpretation is used, which can establish the calculation model of reservoir parameters and the identification criterion of oil and water layer ${ }^{[1]}$. On the basis of the study on the relationship among the lithology, physical property, electrical property, hydrocarbon bearing property and each other, build the interpretation model of the porosity, permeability and saturation by lamination and sub lithology to use the thought of Calibration log data of core analysis data[2-3].

\section{The study of four-property relationship}

The relationship of the lithology, physical property, electrical property, oiliness and between each other is called the four-property relationship of the reservoir [4]. The four-property relationship of the

a Correspondence author : 601159963@qq.com 
reservoir is an important basis for the interpretation of the oil-water layer and the evaluation of reservoir [5].

\subsection{The relationship between lithology and physical property}

The relationship between the lithology and the property is the most important of the four-property relationship, and both of them control the performance of reservoir directly[6], which can be used to judge whether the reservoir performance is good or not.

From Figure 1, shows that the porosity is from $10 \%$ to $30 \%$, the permeability is from $0.8 \times 10^{-3} \mathrm{~mm}^{2}$ to $1000 \times 10^{-3} \mathrm{~mm}^{2}$ of the sand rock in generally, except the argillaceous siltstone.

According to log comprehensive interpretation, lithology and testing, production test information, the corresponding relationship between the porosity and permeability of the different rocks(see table 1) shows that the porosity is mainly concentrated in $5 \% \sim 25 \%$. The permeability distribution has a wider range $\left(0.002 \times 10^{-3} \sim 1000 \times 10^{-3} \mu \mathrm{m}^{2}\right)$, which are mainly distributed in $(0.006 \sim 100) \times 10^{-3} \mu \mathrm{m}^{2}$.

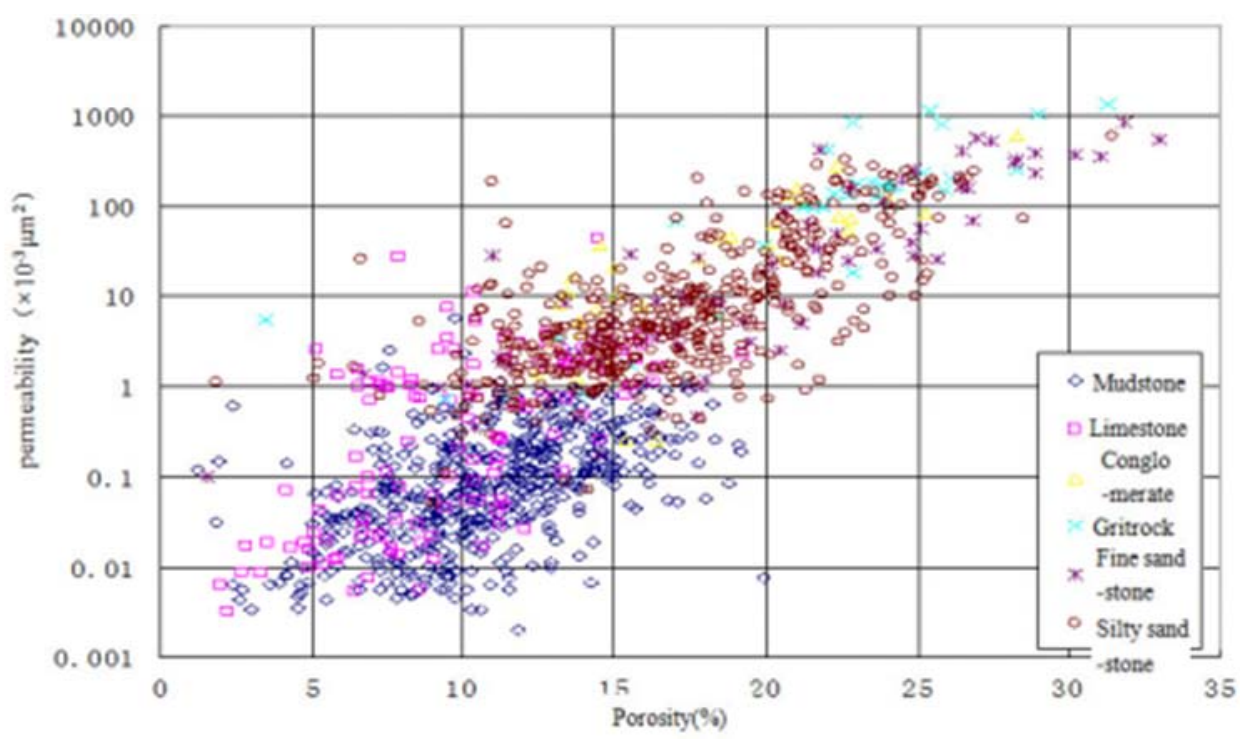

Figure 1. The relationship between physical property and lithology in XN3-21-3 Well (The whole well)

Table1. The statistics table of the porosity and permeability in XN3-21-3 well of Huatugou oilfield

\begin{tabular}{|c|c|c|c|c|c|}
\hline Lithology & $\begin{array}{c}\text { Porosity } \\
/ \%\end{array}$ & $\begin{array}{c}\text { Permeability } \\
/\left(\times 10^{-3} \mu \mathrm{m}^{2}\right)\end{array}$ & Lithology & $\begin{array}{c}\text { Porosity } \\
/ \%\end{array}$ & $\begin{array}{c}\text { Permeability } \\
/\left(\times 10^{-3} \mu \mathrm{m}^{2}\right)\end{array}$ \\
\hline Mudstone & $1 \sim 19$ & $0.003 \sim 8$ & Gritstone & $16 \sim 32$ & $8 \sim 2000$ \\
\hline Limestone & $2 \sim 16$ & $0.03 \sim 10$ & Fine sandstone & $8 \sim 33$ & $0.7 \sim 900$ \\
\hline Conglomerate & $13 \sim 28$ & $9 \sim 800$ & Siltstone & $10 \sim 30$ & $0.8 \sim 1000$ \\
\hline
\end{tabular}

In general, the reservoir rock have more better physical properties of the coarse particles, but the physical property of reservoir is obviously influenced by the type and content of cement. The higher content of cement, the lower porosity and permeability, and the influence of cement content on permeability is greater than the effect on the porosity. The content of carbonate rock has a strong influence on the permeability, but the influence on the porosity is not obvious. In addition, the chink has a certain impact on the property, with the increase of the filler content; the reservoir performance has a trend to go bad. 


\subsection{The relationship between lithology and electrical properties}

In this area, the negative anomaly of the spontaneous potential is changed from large to small when the grain size of reservoir sandstone changed from coarse to fine, while the change of natural gamma value is changed right about. Specifically, the spontaneous potential of the siltstone and sandstone appears negative anomaly obviously, the value of Acoustic time is middle-high, the caliper is drawing down, the value of natural gamma is low, when the clay content increases, the negative abnormal of the spontaneous potential will decrease. When the gray matter is sandwiched in the reservoir, the resistivity and density will increase, while the Acoustic time and the neutron porosity will decrease. When the reservoir contains gravel, the resistivity will increase, and the value of natural gamma will become low.

\subsection{The relationship between lithology, physical property and oil content}

In different clastic rock reservoir, the oil-bearing occurrence in this area is different: the medium and fine sandstone is mainly for oily, the siltstone formation is mainly for oil immersed or grease mark. For the Huatugou oilfield, the oil-bearing of fine sandstone is relatively better, the muddy and limy siltstone is the second, and the limestone as well as the conglomerate is the worst.

If the lithology is the same, the reservoir physical property and oil bearing has a certain correlation, the better the reservoir physical property is, the higher the oil level is. The delta-plain facies, and the delta-front facies of HuaTuGou oilfield what the reservoir has indication of oil and gas appears the oil stains and the grease mark. Through the study on the relationship between physical parameters and oil-bearing in delta-plain facies, delta-front facies and semi-deep lacustrine sub-facies, it is found that there is a positive correlation between permeability and oil saturation, and there is also a positive correlation between porosity and oil content in a certain range.

\subsection{The relationship between electrical properties and oil resistance}

The better the oiliness is, the better the electrical properties is, namely, the higher the resistivity and the greater the Acoustic time are, the smaller the density value and the natural gamma are, the greater the negative difference of spontaneous potential is. The oily limestone has the higher resistance and lower Acoustic time, and the conglomerate has the similar properties, but the resistance is slightly lower. The coring data of Huatugou oilfield confirmed that the more coarse the particle of the rock is, the better the oiliness is. The rock which is saturated by oil or has certain oiliness is fine sandstone mainly, the lithology of rock which has oil stains or grease mark is by siltstone and pelitic siltstone mainly.

The particle size, composition and content of the cement of the reservoir also have some impact on the electrical properties. With the increasing of the particle size, the value of the spontaneous potential, the relative value of gamma ray and the value of acoustic time will increases, but the density will decrease. When the lithology of rock is conglomerate, the value of the acoustic time will decrease. With the increasing of clay content, the value range of spontaneous potential will reduce, the value of gamma ray become more and more big, and the caliper is gradually changed from drawing down to expansion. With the increasing of calcium content, the value of acoustic time will reduce, the value of density becomes larger, the value of microlog shows the top of high value. If the sandstone reservoir is a oil-bearing layer, the value of the deep investigate induction log is greater than the medium investigate induction log, but when it is a water layer, the value of the deep investigate induction log is less than the medium investigate induction log.

\section{The log interpretation model of reservoir parameter}

The reservoir parameter model mainly includes three major aspects, such as lithology, physical property and oiliness[6]. In this paper, give the method of the core scale logging to research the log 
interpretation model.

\subsection{The logging interpretation model of shale content}

The shale content of the reservoir is an important parameter to indicate whether the lithology of the reservoir is good or not. In well logging, it is generally considered that the shale content of the reservoir is mainly composed of clay and fine silt, the particle size is less than or equal to $0.01 \mathrm{~mm}$. As a result of the shale contains more radioactive material, so there is a better reflection in the natural gamma ray and natural gamma ray spectrum curve [7]. It can usually determine the value of clay content in stratum through the grain-size analysis data, and then establish a relationship with well logging curve to calculate the value of the mud content in stratum [8]. In this study, it can establish the relationship between the shaliness (iron soil content, Vsh) and the relative ratio of the spontaneous potential .The core analysis data are acquired from 259 sample which are from 33 different layers in two representative wells, N4-52-3 and N2-38-3. The maximum content of shale content in the sample is $35.4 \%$, the minimum is 0 , and the average is $8.9 \%$. The core analysis of the gravel-bearing sandstone and sandstone reservoir shows that the shale content is closely related to the spontaneous potential. Different well logging methods are selected to calculate the shale content in gravel-bearing sandstone and sandstone reservoir. The shale clay content was calculated by the empirical formula(reference to the other blocks of the Qaidam Basin[11]):

$$
V s h=(S S P-S P S) /(S P M-S P S)
$$

SP correction factor of layer thickness

$$
\alpha=0.9916 /(H-1.598)+0.95
$$

The thickness correction factor of the thickness correction

$$
\text { SSP : } \alpha \times S P
$$

Among them, SP is the value of the spontaneous potential of the target layer $(\mathrm{mV})$, SPM is SP Pure mudstone, SPS is SP Pure sandstone, SP sand: spontaneous potential value of pure sandstone $(\mathrm{mV})$, SP mud: spontaneous potential value of pure mudstone $(\mathrm{mV}), \mathrm{H}$ : bottom depth - top depth $(\mathrm{m})$.

\subsection{The logging interpretation model of the porosity}

The porosity is an important parameter to reflect the physical properties of the reservoir, and it is also one of the necessary parameters for the reserves, productivity calculation and well logging interpretation. It is an important part for reservoir description to use the well logging data to calculate the reservoir porosity, our predecessors have done a lot of related work, put forward many methods for establishing interpretation models of porosity. According to the results of the "Four-property relationship", the correlation between the porosity and the acoustic time is better in the delta-plain facies. There is a high accuracy in the model of the lithology porosity and the acoustic time. With the increasing of the acoustic time, the porosity will increase, namely they are positively correlated (Figure 2). 


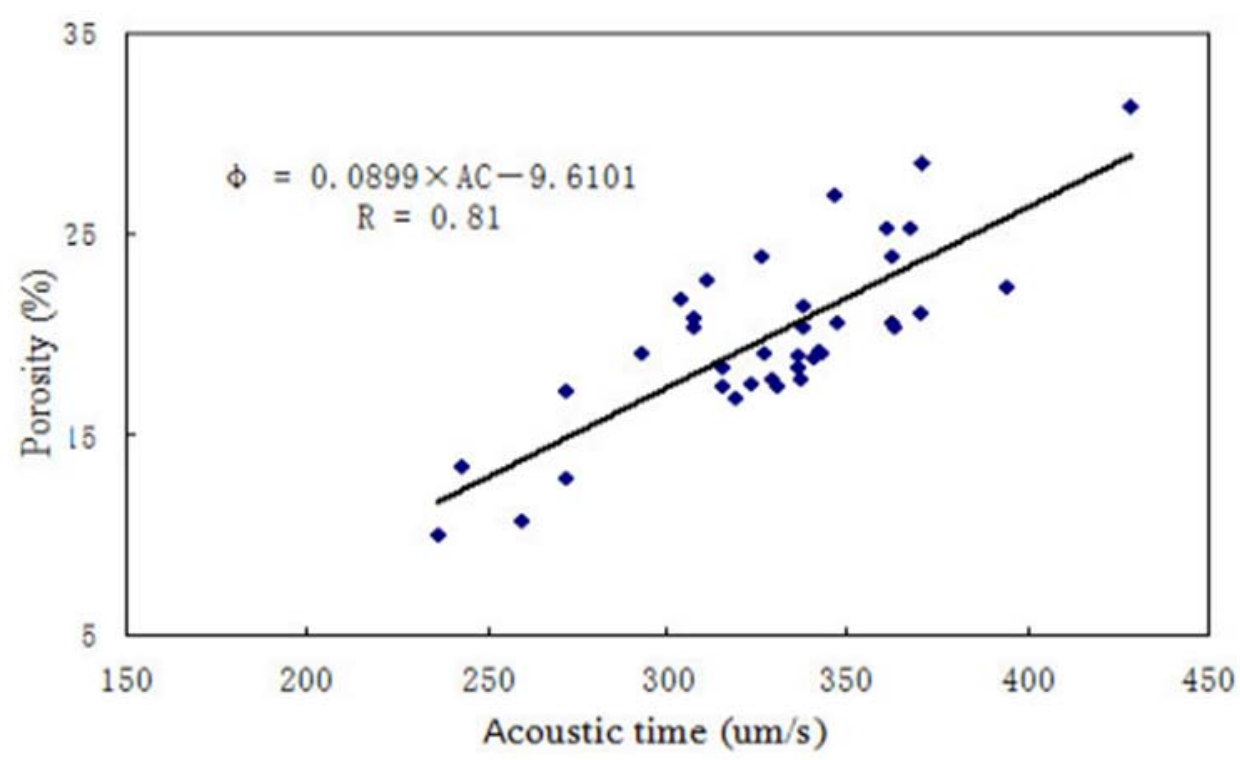

Figure 2. The porosity chart of the delta-plain facies (I-III oil group, from reference [11], slightly modified)

\subsection{The logging interpretation model of the permeability}

Under a certain differential pressure, the permeability of the reservoir can be characterized as the ability that the fluid pass through the rocks, and its value is mainly affected by some factors such as the rock grain size, particle sorting, pore curvature, radius of the pore throat, fluid properties and the distribution form of clay. This impaction can be summarized as the following two points: the first is the radius of the pore throat, which is dependent on the average particle size and the particle sorting of rock; the second is the connectivity between the pores of rock particles, which is dependent on the properties and the content of cement [9-10].

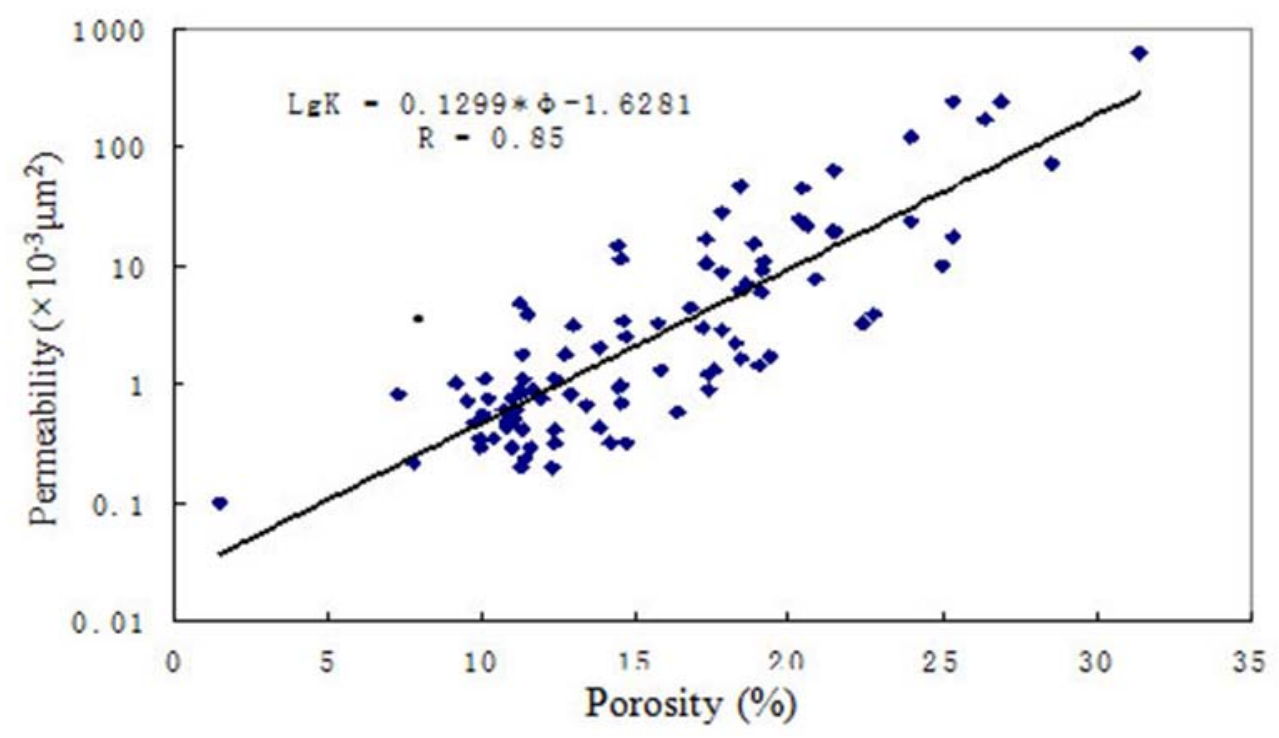

Figure 3. The permeability chart of the delta-plain facies (I-III oil group, from reference [11], slightly modified)

From Figure 3, there is a good correlation between porosity and permeability in I-III, they show 
the exponential relationship. So ues the method of regression to get the permeability of sandstone model, which is:

$$
\operatorname{LgK}=0.1299 \times \varphi-1.6281 \quad R=0.85
$$

\subsection{The logging interpretation model of the oil saturation}

There is a close relationship between lithology and oiliness in this area, which can be seen from the analysis of the relationship between lithology, physical property and oil content. The oil saturation is calculated by the Archie formula because there have no closed coring well in this area. Due to the resistivity of formation water in this area has a certain relationship only with the depth, so water analysis data and depth were selected to build a relation chart to calculate the resistivity of formation water. By means of regression, achieve the relationship between the resistivity of formation water and the depth, which is shown by Fig 4.

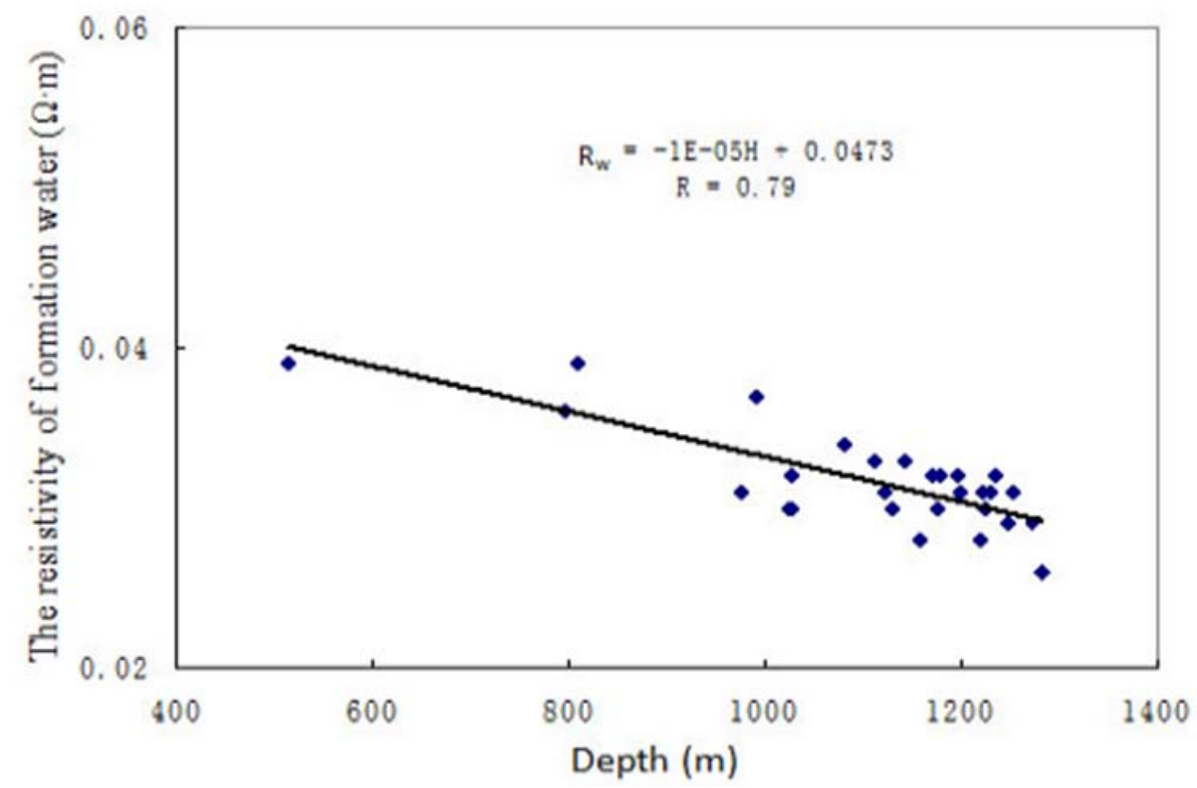

Figure 4. Relationship between the resistivity of formation water and the depth

The total mineralization of formation water has a little change from the upper and lower part in the Huatugou Oilfield, so it do not need to segment when the values of $\mathrm{m}, \mathrm{n}, \mathrm{a}, \mathrm{b}$ should be determined. According to the analysis data of the lithology and electricity, the values of these four parameters are: $\mathrm{a}=1, \mathrm{~b}=1.0177, \mathrm{n}=1.6839$ and $\mathrm{m}=5.4439{ }+0.9253$. For pure sand, substitute these parameters into the Archie formula, then use the relationship between water and oil saturation, water saturation formula for the Huatugou Oilfield can be calculated by formula (5), which is

$$
S w=\left[1.0177 \times R w / \varphi^{(5.4439 \times \varphi+0.9253)} R t\right]^{1 / 6839}
$$

In these formulas, $\mathrm{Sw}$ is water saturation. Rw is the resistivity rate of formation water $\Omega \cdot \mathrm{m}$; Rt is rock resistivity with 100 percent of water, $\Omega \cdot \mathrm{m}, \varphi$ is porosity.

\section{Application of the well logging interpretation results}

Applying these well logging interpretation model, this test use the logging interpretation software to 
give the fine interpretation from I to $\mathrm{X}$ oil group in Huatugou oilfield, which has the good logging information. The interpretation parameters were unified and the interpretation accuracy of oil layer was improved. The oil-water boundary was further distinguished and the reserves was calculated accurately. After recalculation, the geological reserves change of the oil groups are shown in table 2 .

Table 2. Comparison of reserves between 2001 and 2016

\begin{tabular}{|c|c|c|c|c|c|c|}
\hline \multirow{2}{*}{ Oil group } & \multicolumn{3}{|c|}{2001 reserves $\left(\times 10^{4} \mathrm{t}\right)$} & \multicolumn{3}{|c|}{2016 reserves $\left(\times 10^{4} \mathrm{t}\right)$} \\
\hline & $\begin{array}{l}\text { Sandstone } \\
\text { (Class I) }\end{array}$ & $\begin{array}{l}\text { Sandstone } \\
\text { (Class II) }\end{array}$ & Limestone & $\begin{array}{l}\text { Sandstone } \\
\text { (Class I) }\end{array}$ & $\begin{array}{l}\text { Sandstone } \\
\text { (Class II) }\end{array}$ & Limestone \\
\hline I & 304.8 & 17.73 & & 293.7 & \multirow{11}{*}{312.35} & \\
\hline II & 478.8 & 36.11 & & 493.67 & & \\
\hline III & 318.8 & 15.71 & 1.9 & 329.09 & & \\
\hline IV & 267.8 & 37.31 & 3.5 & 360.86 & & 3.44 \\
\hline V & 279.8 & 25.91 & 13.1 & 313.98 & & 1.77 \\
\hline VI & 281.9 & 30.63 & 66.5 & 301.62 & & 26.87 \\
\hline VII & 275.8 & 53.17 & 9.4 & 250.11 & & 8.41 \\
\hline VIII & 59.9 & 16.75 & 2.7 & 65.83 & & \\
\hline IX & 36.69 & 18.81 & 0.8 & 50.87 & & \\
\hline $\mathrm{X}$ & 36.3 & 5.89 & 0.2 & 43.78 & & \\
\hline Subtotal & 2340.6 & 258 & 97.9 & 2503.5 & & 40.49 \\
\hline Total & \multicolumn{3}{|c|}{2696.5} & \multicolumn{3}{|c|}{2856.3} \\
\hline
\end{tabular}

\section{Conclusion and suggestion}

(1) Give an accurate study in the four-property relationship of XN3-21-3 well in HuaTuGou field, and the four-property relationship of the sandstone, fine sandstone and gravel bearing sandstone were analyzed. The foundation was can be built for establishing the reservoir parameter interpretation model.

(2) Research suggests that building the interpretation model hierarchically has a high precision through setting a logging interpretation model of shale content, porosity, permeability and water saturation for the destination layer.

(3) Applying an interpretation model, provide the accurate geological parameters for reservoir simulation and reservoir evaluation as well as a reference for tapping the potential of remaining oil in this area.

\section{Reference}

1. Tao Jiali, Qin Qirong, Lin Jun, et al. Fine log interpretation model of $J_{1} b^{5}$ reservoir in Karamay Oilfield. J. Fault-Block Oil \& Gas Field, 20(5),597-601 (2013)

2. Li Hao, Sun Bing, Wei Xiuping. A study on the log interpretation in volcanic reservoir in the southern Songhuajiang Gas Field. J. Progress in Geophysics, 27(5),203-205 (2012)

3. Li Li, Sun Yaoting, Chen Jian. Well logging interpretation method for Feixianguan and Changxing Formations in Puguang Gas Field. J. Fault-blcok Oil-gas Field, 18(4),508-511 (2011) 
4. Li Jinzhu, Liu Zong, Lu Chunhui, Li Airun. Study on the Relationship of Four Characteristics in Dongzhuang Reservoir. J. Oil-GASFIELD SURFACE ENGINEERING, 30(4),24-25 (2011)

5. Chen Xiaofang, Zhang Xiaoli. Study on four-proporty Relationship of Chang 6 Reservoir in Nanniwan Oilfield. J. SCI-TECH INFORMATION DEVELOPMENT \& ECONOMY, 20(9), 163-166 (2010)

6. Yue Shaofei, Liu Jie, Ma Lina, Zhou Xuefeng. The four-property relationship of the reservoir and the standard of effective thickness lower limit in the third member of Liushagang formation. J. Petroleum Geology and Recovery Efficiency, 20(4),42-45+113-114 (2013)

7. Zhou Liangzhi, Xie Ranhong. Application of Core Calibration Logging in Establishing Log Interpretation Model. J. JOURNAL OF OIL AND GAS TECHNOLOGY, 33(5),99-102 (2011)

8. Wan Zhoujuan, Guo Kaiming. Study on fine logging interpretation model of Jin 16 area in Liaohe Oilfield. J. Overseas well logging technology,(4),25-29 (2013)

9. Zhai ZhongXi, Tai Huaizhong, Zhou Gaiying. Study on the interpretation method of Xinzhuang oilfield heavy oil well logging. J. Journal of Jianghan Petroleum Institute, 25(4), 61 (2003)

10. Zhang Liyan, Cai Juhong, Chen Ganghua. Methods for Interpretating Oil-bearing Property of Glutenite Reservoir. J. WLT, 26(2),134-136 (2002)

11. Zhimeng Zhao,Yunbin Xiong. Fine log interpretation of structural reservoir and modeling study in Huatugou Oilfield. R. Qinghai Exploration and development research institute of Qinghai oilfield branch company (2006) 